\title{
Traumatic Abdominal Solid Organ Injury Patients Might Benefit From Thromboelastography-Guided Blood Component Therapy
}

\author{
Hao Wang ${ }^{\mathrm{a}, \mathrm{d}}$, Richard D. Robinson ${ }^{\mathrm{a}}$, Jessica L. Phillips ${ }^{\mathrm{b}}$, Andrew Ryon ${ }^{\mathrm{a}}$, Scott Simpson ${ }^{\mathrm{a}}$, \\ Jonathan R. Ford ${ }^{\mathrm{a}}$, Johnbosco Umejiego ${ }^{\mathrm{b}}$, Therese M. Duane ${ }^{\mathrm{c}}$, Bradley Puttyc, \\ Nestor R. Zenarosa ${ }^{\mathrm{a}}$
}

\begin{abstract}
Background: Thromboelastography (TEG) has been utilized for the guidance of blood component therapy (BCT). We aimed to investigate the association between emergent TEG-guided BCT and clinical outcomes in patients with traumatic abdominal solid organ (liver and/ or spleen) injuries.
\end{abstract}

Methods: A single center retrospective study of patients who sustained traumatic liver and/or spleen injuries receiving emergent BCT was conducted. TEG was ordered in all these patients. Patient demographics, general injury information, outcomes, BCT, and TEG parameters were analyzed and compared in patients receiving TEGguided BCT versus those without.

Results: A total of 166 patients were enrolled, of whom 52\% (86/166) received TEG-guided BCT. A mortality of $12 \%$ was noted among patients with TEG-guided BCT when compared with $19 \%$ of mortality in patients with non-TEG-guided BCT $(\mathrm{P}>0.05)$. An average of 4 units of packed red blood cell (PRBC) was received in patients with TEG-guided BCT when compared to an average of 9 units of PRBC received in non-TEG-guided $\mathrm{BCT}$ patients $(\mathrm{P}<0.01)$. A longer hospital length of stay (LOS, $19 \pm 16$ days) was found among non-TEGguided BCT patients when compared to the TEG-guided BCT group $(14 \pm 12$ days, $\mathrm{P}<0.05)$. TEG-guided BCT showed as an independent factor associated with hospital LOS after other variables were adjusted (coefficiency: 5.44, 95\% confidence interval: 0.69 - 10.18).

Conclusions: Traumatic abdominal solid organ injury patients re-

Manuscript accepted for publication March 17, 2017

aDepartment of Emergency Medicine, Integrative Emergency Services Physician Group, John Peter Smith Health Network, 1500 S. Main St., Fort Worth, TX 76104, USA

${ }^{b}$ Research Institute, John Peter Smith Health Network, 1500 S. Main St., Fort Worth, TX 76104, USA

'Department of General Surgery, John Peter Smith Health Network, 1500 S. Main St., Fort Worth, TX 76104, USA

${ }^{\mathrm{d} C o r r e s p o n d i n g ~ A u t h o r: ~ H a o ~ W a n g, ~ D e p a r t m e n t ~ o f ~ E m e r g e n c y ~ M e d i c i n e, ~}$ Integrative Emergency Services, John Peter Smith Health Network, 1500 S. Main St., Fort Worth, TX 76104, USA. Email: hwang01@jpshealth.org

doi: https://doi.org/10.14740/jocmr3005w ceiving blood transfusions might benefit from TEG-guided BCT as indicated by less blood products needed and less hospitalization stay among the cohort.

Keywords: Traumatic liver and/or spleen injury; Thromboelastography; Blood component therapy

\section{Introduction}

In recent years, trauma-induced coagulopathy (TIC) has been recognized in patients experiencing severe traumatic injuries. Relatively higher mortality and morbidity rates were observed in this group [1-3]. Currently, the severe trauma management timeline requires initiation of a more balanced blood component therapy (BCT) process as soon as possible. Improved survival rates were reported when these interventions were employed early $[4,5]$.

Thromboelastography (TEG), a method of detecting blood coagulation efficiency, has recently been recognized as a more sensitive test than traditional coagulation tests (i.e., prothrombin time (PT), partial thromboplastin time (PTT), and international normalized ratio (INR)) to determine TIC, guide BCT, and predict outcomes [6-9]. Several TEG parameters can be reported during the early trauma resuscitation phase including reaction time (R), coagulation time (K), $\alpha$ angle $(\mathrm{A})$, and maximal amplitude (MA). Abnormal TEG values indicate patients with a higher chance of abnormal post-injury bleeding [10, 11]. More importantly, specific TEG parameters further guide specific blood component transfusion needs. In general, prolonged $\mathrm{R}$ values indicate additional fresh frozen plasma (FFP) requirements, prolonged $\mathrm{K}$ or reduced $\mathrm{A}$ values indicate additional cryoprecipitate requirements, while reduced MA values indicate additional platelet transfusion requirements [12]. TEG is used in the early phase of severe trauma damage control resuscitation, especially among patients requiring blood transfusions where optimizing BCT is of paramount interest $[13,14]$.

It is well known that the liver and spleen play important roles in the homeostasis of coagulation pathways $[15,16]$. Traumatic liver and/or spleen injuries could significantly affect coagulopathy. The usefulness of TEG-guided BCT in these patients and whether TEG can predict patient hospital 
outcomes after initial BCT are clinically relevant questions $[17,18]$. To better understand the application of TEG during early trauma resuscitation in patients with traumatic liver and/ or spleen injuries, we aimed to determine the role of TEG as a guide for emergent $\mathrm{BCT}$ and its association with patient clinical outcomes.

\section{Materials and Methods}

\section{Study design and participation}

We performed a single center, secondary data analysis using observational data from a local level I trauma registry covering the period of June 2013 through December 2015. All study data were prospectively collected with the exception of TEG results and transfused blood type and amount, which were manually abstracted by study investigators. To confirm data accuracy, all manually collected data were validated between two investigators and discrepant data were validated by a third. All patients registered in the local trauma registry were initially reviewed.

Inclusion criteria were: 1) patients who sustained traumatic liver and/or spleen injuries; 2) patients who received any types of blood products within the first $24 \mathrm{~h}$ of hospital arrival; and 3) TEG performed either before the blood transfusion or during the emergent resuscitations at operating room or emergency department (ED) which were collected within the first 2 $\mathrm{h}$ of hospital arrival.

Exclusion criteria were: 1) patients with no CT evidence of liver and/or spleen injuries/lacerations; 2) patients with no surgical evidence of liver and/or spleen injuries per operative reports; 3) deceased patients with no evidence of liver and/or spleen injuries; 4) patients with liver and/or spleen injuries not related to acute traumatic events occurring within $72 \mathrm{~h}$ prior to hospital admission; 5) patients transferred from other facilities with unknown status of blood transfusion prior to arrival at the study institution; 6) no TEG preformed within the first $2 \mathrm{~h}$ of hospital arrival; and 7) patients exposed to anticoagulants were also excluded as emergent platelet mapping, including inhibition of platelets to arachidonic acid and adenosine diphosphate aggregation studies, were not available during the early trauma resuscitation phase at the study institution. A statistically insignificant number of patients fell into this subgroup of anticoagulant exposed patients.

\section{Variables}

Four TEG parameters were tested in this study, reaction time (R-time), speed of clot formation time (K-time), $\alpha$ angle (A), and MA. The normal ranges of these parameters are: R-time (2.5 - $10 \mathrm{~min})$, K-time (1 - $3 \mathrm{~min}), \mathrm{A}\left(53-72^{\circ}\right)$, and MA (50 - $70 \mathrm{~mm})$. Any results outside of these ranges were considered abnormal. TEG-guided BCT group was defined as those patients receiving different blood component transfusions that matched all the following guidelines: 1) FFP given when prolonged R-time(s) resulted; 2) cryoprecipitate given when prolonged K-time(s) or reduced A(s) resulted; 3) platelets given when reduced MA(s) resulted. Few patients received anti-fibrinolytics in this study and LY30 (percent clot lysis at $30 \mathrm{~min}$ ) was not investigated as it was not included as an early TEG reported item. Patients whose care did not follow the above transfusion guideline or patients who received unnecessary blood components based on the TEG results were placed in the non-TEG-guided group. Mortality was defined as in-hospital all-cause mortality.

\section{Study protocol}

To determine the role of TEG-guided BCT, patients were divided into two groups (TEG-guided versus non-TEG-guided). General patient characteristics (age, sex, race, and mode of arrival), clinical variables (initial vital signs, type of injury, and severity of injury), study lab values (TEG parameters including R-time, K-time, angle, and maximal amplitude), blood component transfusions (packed red blood cells (PRBC), fresh frozen plasma (FFP), platelets, and cryoprecipitate), and outcomes (length of stay (LOS) and in-hospital mortality) were analyzed and compared among these two groups. Specifically, to avoid the confounding factors, potential factors (initial vital signs, severity of injury, age, initial TEG value, and TEG-guided BCT) affecting patient LOS were analyzed together using multivariate linear regression analysis. This regression model was again tested to minimize variable selection and collinearity biases. This study was approved by the local institutional review board.

\section{Statistical analysis}

Pearson's Chi-square $\left(\chi^{2}\right)$ analysis was used to analyze differences in relative frequencies among groups for categorical variables. Student's $t$-tests and Wilcoxon rank-sum (MannWhitney) test were used to test differences between groups for continuous variables. Multivariate linear regression model included adjustments for all factors potentially predicting hospital LOS. Variance inflation factor (VIF) was used for assessing the severity of multi-collinearity in the regression model and those with high VIF $(>10)$ were considered as having collinearity. A Ramsey regression equation specification error test (RESET) was performed to test regression model specification and determine whether one or more relevant variables were omitted from the model. A P value more than 0.05 was considered no significant omitted variable bias and model specification errors did not substantially affect the estimate of regression coefficients. All descriptive and statistical analyses were performed using Stata 12.0 (College Station, TX). A P value less than 0.05 was considered statistically significant.

\section{Results}

During the period of June 2012 through December 2015, a total of 474 patients sustaining traumatic liver and/or spleen injuries were enrolled in the local trauma registry. TEG was performed 
Table 1. Comparisons Between TEG Versus Non-TEG-Guided Blood Component Transfusion Among Liver and Spleen Injury Patients

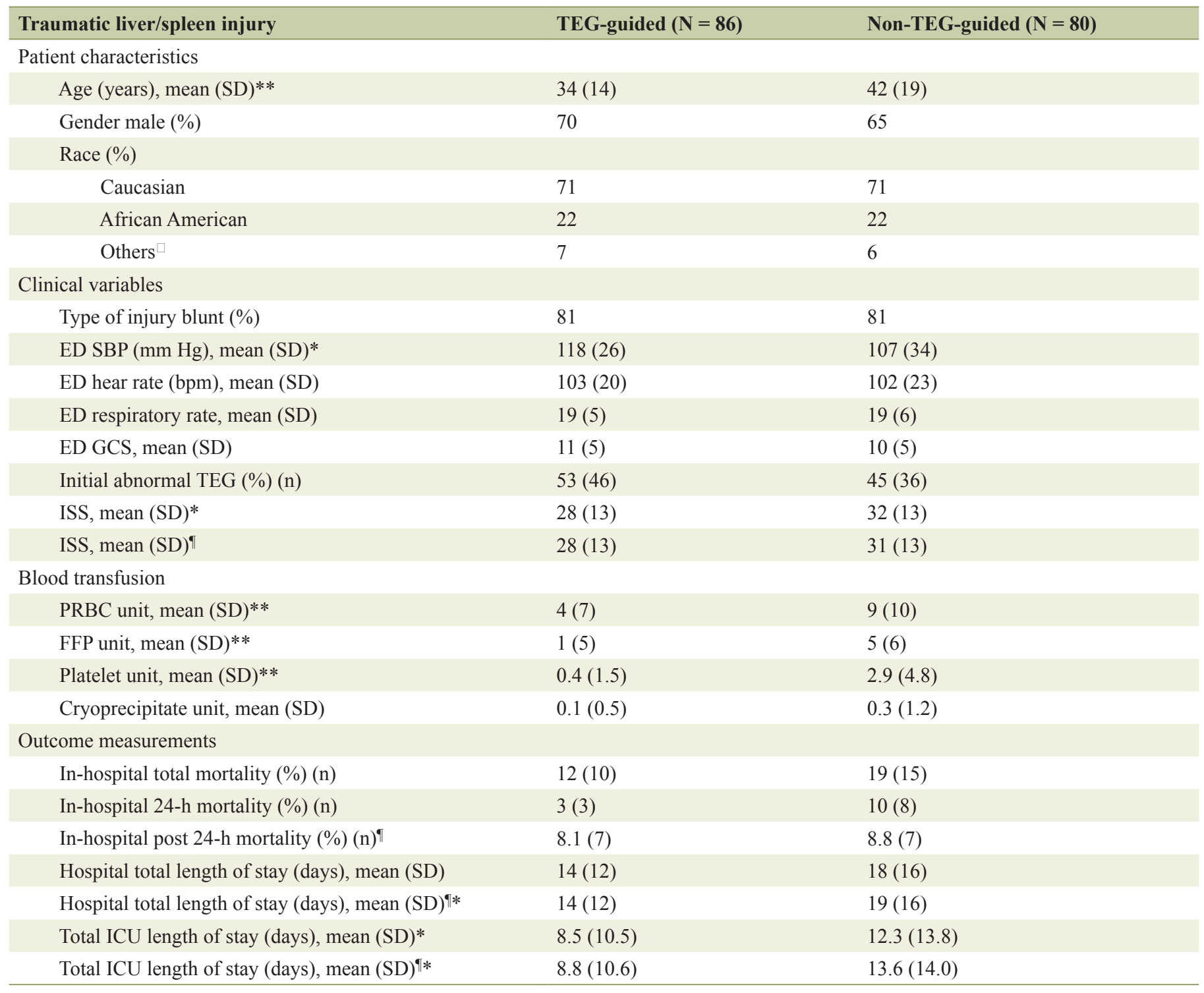

SD: standard deviation; N: number; ED: emergency department; SBP: systolic blood pressure; GCS: Glasgow coma scale; ISS: injury severity scale; PRBC: packed red blood cell; FFP: fresh frozen plasma; TEG: thromboelastography; ICU: intensive care unit. Others in race: include Asian, Pacific Islander, Hispanic, or unknown. ${ }^{* *} \mathrm{P}<0.01$; ${ }^{*} \mathrm{P}<0.05$. TStudy excluded patients who died within the first $24 \mathrm{~h}$ of hospital arrival.

within the first $2 \mathrm{~h}$ of hospital arrival in $76 \%(360 / 474)$ of the study patients. Among those for which TEG was performed, $46 \%(166 / 360)$ received blood transfusions within the first 24 $\mathrm{h}$ of hospital arrival. Therefore, a total of 166 patients were included in the final analysis.

Analysis was focused on TEG-guided BCT. Patients whose $\mathrm{BCT}$ was strictly managed using TEG results were placed into the TEG-guided BCT group. Those whose BCT was not strictly managed using TEG results were placed into the non-TEGguided group. Patients in non-TEG-guided group tended to be older with relatively lower initial ED systolic blood pressure. Patients in non-TEG-guided group also showed more severe level of injury (injury severity scale (ISS)) than those without.
This became less significant if we excluded patients who died within the first $24 \mathrm{~h}$ of hospital arrival (Table 1). No significant differences were noted for patient gender, race, type of injury, GCS, other vital signs (including ED heart rate and respiratory rate), and initial abnormal TEG results between two groups. However, patients who followed TEG guidance tended to receive significantly less amounts of BCT than those without (Table 1, P < 0.01). In general, in-hospital mortality was not changed significantly regardless of whether patients followed TEG-guided BCT.

Since TEG did not affect mortality, we performed further data analysis and excluded patients who died within the first $24 \mathrm{~h}$ of hospital arrivals. Our results found that significantly 
Table 2. Factors Associated With Hospital Length of Stay Using Multivariate Linear Regression Analysis $\pi$

\begin{tabular}{lllll}
\hline Factors & Coefficiency & $\mathbf{9 5 \%}$ confidence interval & P value & VIF \\
\hline Age & -0.11 & -0.25 to 0.04 & 0.14 & 1.14 \\
Initial ED SBP & -0.06 & -0.14 to 0.02 & 0.16 & 1.12 \\
Initial ED GCS & -0.30 & -0.77 to 0.16 & 0.20 & 1.18 \\
Initial abnormal TEG results & -1.85 & -6.44 to 2.75 & 0.43 & 1.07 \\
Injury severity scale (ISS) & 0.03 & -0.16 to 0.23 & 0.74 & 1.27 \\
Non-TEG-guided BCT & 5.44 & 0.69 to 10.18 & 0.03 & 1.13 \\
\hline
\end{tabular}

Ramsey RESET: P = 0.09. ED: emergency department; SBP: systolic bood pressure; GCS: Glasgow coma scale; ISS: injury severity scale; TEG: thromboelastography; VIF: variance inflation factor; RESET: regression equation specification error test. "Study excluded patients who died within the first $24 \mathrm{~h}$ of hospital arrival.

prolonged hospital and ICU LOS were noted among patients in the non-TEG-guided group when compared with patients in the TEG-guided group (Table 1). Whereas, no significant differences of patient injury severity (ISS) were found between patients of two groups who survived longer than the first $24 \mathrm{~h}$ of hospital stay.

To avoid the confounding factors, a multivariate linear regression was performed and hospital LOS showed a significant increasing pattern in patients with non-TEG-guided BCT. More specifically, TEG-guided BCT was considered as an independent factor predicting hospital LOS (Table 2, $\mathrm{P}<0.05$ ). Meanwhile, there were no indications of collinearity (VIF < 10) nor omitted variable bias (Ramsey RESET, $P>0.05$ ) in this regression model (Table 2).

\section{Discussion}

In recent years, studies showed optimal patient care outcomes when damage control resuscitation (DCR) has been emphasized in severe trauma [19]. TEG has been used more often during DCR to determine patient need for BCT [20]. Our study showed that patients who followed TEG-guided BCT tended to receive significant less amounts of blood products with similar mortality and less hospital or ICU stay when compared with non-TEG-guided patients. Additionally, TEG-guided $\mathrm{BCT}$ was considered as an independent factor associated with patient LOS with minimal bias in a risk adjusted model. Given the fact that TEG-guided BCT reported more on patients receiving massive transfusion and little is known of its benefit on patients with liver and/or spleen injuries receiving blood transfusions in general, the results of this study add evidence to the literature pool in terms of expanding the use of TEGguided $\mathrm{BCT}$ and recognizing its patient care outcome benefit indicated as less blood products needed and shortened hospital LOS among the cohort.

Previous studies reported TEG predicts hospital mortality $[9,21]$. However, many studies mainly focused on patient receiving massive transfusion and did not fully determine whether mortality linked to the severity of TIC or to the initiation of appropriate BCT $[14,22]$. Since initial abnormal TEG can be corrected after bleeding controlled and/or proper BCT, therefore we believe the value of TEG is to guide BCT and monitor post-transfusion response instead of predicting patient care outcomes. On the other hand, TEG was reported in other studies as a better marker for early recognizing TIC and could be recognized as a marker for initiating BCT [8, 12, 20, 23]. Therefore, this study was mainly focused on determining the potential benefits among patients with TEG-guided BCT.

We found relatively high mortality rate among patients with non-TEG-guided BCT. Due to relatively small sample size, our study did not reach statistically significant differences. However, this study expanded TEG-guided BCT to all patients receiving blood transfusions which could have this discrepancy due to different patient population selections. More importantly, apart from patients died within the first $24 \mathrm{~h}$ of hospital arrival, an average of 5-day longer hospitalizations occurred in patients with non-TEG-guided group. This might be due to less significant amounts of blood product received when patient strictly followed TEG guidance. It has been reported more blood product transfusions lead to severe inhospital complications (such as adult respiratory distress syndrome, acute lung injury, congestive heart failure, etc.) which might be related to prolonged patient hospitalizations [24, 25]. Other reports also found less blood products transfused among patients with TEG-guided BCT than those without $[14,26]$. Our study results were consistent with those previous findings.

This seems to be the huge benefit among patients receiving TEG-guided BCT if other biases proved to be minimized. Since significant amounts of risk factors including, but not limited to, severity of trauma injury, hospital complications, age, and patient comorbidities, could potentially affect patient LOS, a risk adjusted model was built in this study to minimize these biases. We included trauma severity (ISS), age, initial abnormal TEG results, and whether TEG-guided BCT followed as potential factors affecting patient LOS. We also include initial GCS as a potential factor considering lower GCS could be associated with potential traumatic brain injuries. Though not all potential risks were completely included in the study risk adjusted model, this model was proved to be sufficient to identify TEG-guided BCT as an independent factor associated with patient LOS.

Overall, TEG is valuable in guiding blood transfusions with respect to specific component needs. Less amounts of blood products might be required if TEG-guided BCT strictly followed which subsequently leads to shorted hospitalizations. However, its mechanism(s) are still not fully clear. More research is needed to explore whether TEG predicts response to 
treatment, whether TEG-guided BCT minimizes transfusionassociated complications, and whether TEG-guided BCT could be recommended to all traumatic patients receiving blood transfusions.

\section{Limitations}

This is a secondary data analysis which cannot demonstrate causality due to limited information accuracy, missing data, and potential selection bias. Due to the study's small sample size, statistically significant differences between variables cannot be reached when comparing groups - a limitation warranting future investigation with larger sample sizes. Patients with traumatic liver and/or spleen injuries who expired during hospitalization may be the result of other injuries sustained during the sentinel trauma event. Assuming patient's true severity of injury based on ISS might not be accurate from one patient to the next. This study focused on BCT within the first $24 \mathrm{~h}$ of hospitalization. We are unable to determine the benefit of TEG-guided BCT after the initial $24 \mathrm{~h}$ of hospitalization. Other factors (such as patient hospital complications, comorbidities) that potentially affect hospitalizations were not included in the multivariate linear regression model. We did not analyze all TEG result parameters (such as LY30, EPL, inhibition of platelets to arachidonic acid, adenosine diphosphate aggregation) as the percent of the study sample who received anti-fibrinolytics was very low. We are unable to determine the value of TEG in guiding BCT among patients exposed to anticoagulants (such as platelet antagonists or new oral anticoagulants) nor did we have enough data to determine BCT superiority to massive transfusion protocol with fixed ratio blood component transfusions. Additionally, only four different blood component transfusions were investigated in this study. In summary, these limitations warrant further examination in multicenter, prospective studies with larger sample sizes.

\section{Conclusions}

Traumatic abdominal solid organ (liver and/or spleen) injury patients receiving blood transfusions might benefit from TEG-guided BCT indicated by less blood products needed and shortened hospital LOS among the cohort.

\section{Source(s) of Support}

None.

\section{References}

1. Brohi K, Singh J, Heron M, Coats T. Acute traumatic coagulopathy. J Trauma. 2003;54(6):1127-1130.

2. MacLeod JB, Lynn M, McKenney MG, Cohn SM, Murtha M. Early coagulopathy predicts mortality in trauma. J
Trauma. 2003;55(1):39-44

3. Maegele M, Lefering R, Yucel N, Tjardes T, Rixen D, Paffrath T, Simanski C, et al. Early coagulopathy in multiple injury: an analysis from the German Trauma Registry on 8724 patients. Injury. 2007;38(3):298-304.

4. Cinat ME, Wallace WC, Nastanski F, West J, Sloan S, Ocariz J, Wilson SE. Improved survival following massive transfusion in patients who have undergone trauma. Arch Surg. 1999;134(9):964-968; discussion 968-970.

5. Riskin DJ, Tsai TC, Riskin L, Hernandez-Boussard T, Purtill M, Maggio PM, Spain DA, et al. Massive transfusion protocols: the role of aggressive resuscitation versus product ratio in mortality reduction. J Am Coll Surg. 2009;209(2):198-205.

6. Hagemo JS, Christiaans SC, Stanworth SJ, Brohi K, Johansson PI, Goslings JC, Naess PA, et al. Detection of acute traumatic coagulopathy and massive transfusion requirements by means of rotational thromboelastometry: an international prospective validation study. Crit Care. 2015;19:97.

7. Kane I, Ong A, Orozco FR, Post ZD, Austin LS, Radcliff KE. Thromboelastography predictive of death in trauma patients. Orthop Surg. 2015;7(1):26-30.

8. Holcomb JB, Minei KM, Scerbo ML, Radwan ZA, Wade CE, Kozar RA, Gill BS, et al. Admission rapid thrombelastography can replace conventional coagulation tests in the emergency department: experience with 1974 consecutive trauma patients. Ann Surg. 2012;256(3):476-486.

9. Carroll RC, Craft RM, Langdon RJ, Clanton CR, Snider CC, Wellons DD, Dakin PA, et al. Early evaluation of acute traumatic coagulopathy by thrombelastography. Transl Res. 2009;154(1):34-39.

10. Liou DZ, Shafi H, Bloom MB, Chung R, Ley EJ, Salim A, Tcherniantchouk O, et al. Defining early traumainduced coagulopathy using thromboelastography. Am Surg. 2014;80(10):994-998.

11. Lier H, Bottiger BW, Hinkelbein J, Krep H, Bernhard M. Coagulation management in multiple trauma: a systematic review. Intensive Care Med. 2011;37(4):572-582.

12. Walsh M, Thomas SG, Howard JC, Evans E, Guyer K, Medvecz A, Swearingen A, et al. Blood component therapy in trauma guided with the utilization of the perfusionist and thromboelastography. J Extra Corpor Technol. 2011;43(3):162-167.

13. Mahambrey T, Pendry K, Nee A, Bonney S, Nee PA. Critical care in emergency department: massive haemorrhage in trauma. Emerg Med J. 2013;30(1):9-14.

14. Kashuk JL, Moore EE, Wohlauer M, Johnson JL, Pezold M, Lawrence J, Biffl WL, et al. Initial experiences with point-of-care rapid thrombelastography for management of life-threatening postinjury coagulopathy. Transfusion. 2012;52(1):23-33.

15. Zare F, Magnusson M, Mollers LN, Jin T, Tarkowski A, Bokarewa M. Single-stranded polyinosinic acid oligonucleotides trigger leukocyte production of proteins belonging to fibrinolytic and coagulation cascades. J Leukoc Biol. 2008;84(3):741-747.

16. Mackavey CL, Hanks R. Hemostasis, coagulation abnormalities, and liver disease. Crit Care Nurs Clin North Am. 
2013;25(4):435-446, v.

17. Stravitz RT, Lisman T, Luketic VA, Sterling RK, Puri P, Fuchs M, Ibrahim A, et al. Minimal effects of acute liver injury/acute liver failure on hemostasis as assessed by thromboelastography. J Hepatol. 2012;56(1):129-136.

18. Sawhney C, Kaur M, Gupta B, Singh PM, Gupta A, Kumar S, Misra MC. Critical care issues in solid organ injury: Review and experience in a tertiary trauma center. Saudi J Anaesth. 2014;8(Suppl 1):S29-35.

19. Shrestha B, Holcomb JB, Camp EA, Del Junco DJ, Cotton BA, Albarado R, Gill BS, et al. Damage-control resuscitation increases successful nonoperative management rates and survival after severe blunt liver injury. J Trauma Acute Care Surg. 2015;78(2):336-341.

20. Johansson PI, Sorensen AM, Larsen CF, Windelov NA, Stensballe J, Perner A, Rasmussen LS, et al. Low hemorrhage-related mortality in trauma patients in a Level I trauma center employing transfusion packages and early thromboelastography-directed hemostatic resuscitation with plasma and platelets. Transfusion. 2013;53(12):30883099.

21. Park MS, Salinas J, Wade CE, Wang J, Martini W, Pusateri AE, Merrill GA, et al. Combining early coagulation and inflammatory status improves prediction of mortality in burned and nonburned trauma patients. J Trauma. 2008;64(2 Suppl):S188-194.

22. Pezold M, Moore EE, Wohlauer M, Sauaia A, Gonzalez E, Banerjee A, Silliman CC. Viscoelastic clot strength predicts coagulation-related mortality within 15 minutes. Surgery. 2012;151(1):48-54.

23. Tapia NM, Chang A, Norman M, Welsh F, Scott B, Wall MJ, Jr., Mattox KL, et al. TEG-guided resuscitation is superior to standardized MTP resuscitation in massively transfused penetrating trauma patients. J Trauma Acute Care Surg. 2013;74(2):378-385; discussion 385-376.

24. Sihler KC, Napolitano LM. Complications of massive transfusion. Chest. 2010;137(1):209-220.

25. Chan CM, Shorr AF, Perkins JG. Factors associated with acute lung injury in combat casualties receiving massive blood transfusions: a retrospective analysis. J Crit Care. 2012;27(4):419 e417-414.

26. Wikkelso A, Wetterslev J, Moller AM, Afshari A. Thromboelastography (TEG) or thromboelastometry (ROTEM) to monitor haemostatic treatment versus usual care in adults or children with bleeding. Cochrane Database Syst Rev. 2016;(8):CD007871. 